\title{
Estudio exploratorio de los nativos digitales en el ámbito Universitario de la Región Mixteca
}

\section{Exploratory study of digital natives at University in the Mixteca Region}

\author{
ALLENDE-HERNÁNDEZ Olivia†*, REYES-ESPINOZA, Celia Bertha y SÁNCHEZ-PLATAS, \\ Liliana Eneida
}

Universidad Tecnológica de la Mixteca

ID $1^{\mathrm{er}}$ Autor: Olivia, Allende-Hernández, ORC ID: 0000-0002-8528-457X, Researcher ID Thomson: K-1473-2017, CVU CONACYT ID: 338719

ID $1^{\text {er }}$ Coautor: Celia Bertha, Reyes-Espinoza / ORC ID: 0000-0003-3787-912X, Researcher ID Thomson: AAP-12312020, CVU CONACYT ID: 972519

ID $2^{\text {do }}$ Coautor: Liliana Eneida, Sánchez-Platas / ORC ID: 0000-0001-5233-3868, CVU CONACYT ID: 79644

DOI: $10.35429 / J U M .2020 .11 .4 .10 .16$

Recibido 12 de Marzo, 2020; Aceptado 30 Junio, 2020

\section{Resumen}

La problemática sobre el uso eficiente de herramientas tecnológicas por parte de los estudiantes al ingresar al ámbito universitario impacta en mayor medida a los alumnos provenientes de comunidades rurales debido al precedente de falta de accesibilidad de herramientas tecnológicas. Garantizar una educación inclusiva, equitativa y de calidad es uno de los objetivos para lograr el desarrollo sostenible, por lo cual los estudiantes deben tener acceso igualitario a una educación de calidad. En el caso de las zonas rurales no se puede considerar a los alumnos como "Nativos Digitales" solo por haber crecido en una era digital. Sobre la base de la metodología de estudio de caso y teniendo en cuenta las premisas anteriores, en este artículo se ha investigado el nivel de competencia tecnológica de los llamados Nativos Digitales que ingresan a la Universidad Tecnológica de la Mixteca (UTM). Se ha identificado que los estudiantes de nuevo ingreso de la UTM no se consideran a sí mismos como Nativos Digitales y reconocen tener deficiencias en sus conocimientos respecto al uso de herramientas tecnológicas. Además, los alumnos de nuevo ingreso se enfrentan a requerimientos y expectativas académicas para las cuales la mayoría no están preparados. A pesar de las deficiencias, los estudiantes muestran disponibilidad y accesibilidad para aprender a utilizar herramientas tecnológicas que les permitan alcanzar un aprendizaje significativo.

Nativos Digitales, Región Mixteca, Herramientas Tecnológicas

\begin{abstract}
First year university students need to make efficient use of technological tools, but there is a lack of accessibility of these tools to students from rural communities. This is a problem as one of the sustainable development goals is guaranteeing quality education that is both inclusive and equitable for all students. In the case of rural areas, students cannot be considered "Digital Natives" just because they have grown up in the digital age, because they have not had the exposure to technological tools that their urban counterparts have had. Based on the case study methodology and in consideration of the foregoing premises, this article has applied a strategy to investigate the level of technological competence of the so-called Digital Natives entering the Technological University of the Mixteca (UTM). The study identified that first-year UTM students do not consider themselves to be Digital Natives and acknowledge having deficiencies in their knowledge in the use of technological tools. Additionally, incoming students face academic requirements and expectations for which most are not prepared. Despite the deficiencies, students show a disposition and ability to learn to use technological tools that allow them to achieve meaningful learning.
\end{abstract}

Digital Natives, Mixteca Region, Technological Tools

Citación: ALLENDE-HERNÁNDEZ Olivia, REYES-ESPINOZA, Celia Bertha y SÁNCHEZ-PLATAS, Liliana Eneida. Estudio exploratorio de los nativos digitales en el ámbito Universitario de la Región Mixteca. Revista de Gestión Universitaria 2020. 4-11: $10-16$

\footnotetext{
* Correspondencia del Autor (Correo electrónico: oallende@ mixteco.utm.mx)

$\dagger$ Investigador contribuyendo como primer autor.
} 


\section{Introducción}

El contexto de estudio de la presente investigación está suscrito en la Universidad Tecnológica de la Mixteca (UTM), localizada en la Región Baja de la Mixteca Oaxaqueña, una de las regiones con mayor índice de migración, pobreza y rezago educativo (CONEVAL, 2015). Otro factor a considerar es el porcentaje de etnias existentes en la entidad oaxaqueña. De acuerdo con la Comisión Nacional para el Desarrollo de los Pueblos Indígenas (CDI) anteriormente Instituto Nacional Indigenista (INI), en el estado de Oaxaca se encuentran establecidos 18 de los 68 grupos étnicos o naciones sobrevivientes de la civilización del México Antiguo: Mixtecos, Zapotecos, Triquis, Mixes, Chatinos, Chinantecos, Huaves, Mazatecos, Amuzgos, Nahuas, Zoques, Chontales de Oaxaca, Cuicatecos, Ixcatecos, Chocholtecos, Tacuates, Afromestizos de la costa chica y en menor medida Tzotziles; que en conjunto son 1,734,658 habitantes, es decir el $43.7 \%$ de total de la población, distribuidos en 2,563 localidades. (INEGI, 2016).

El Instituto Nacional para la Evaluación de la Educación (INEE, 2019) indica que en Oaxaca desde la educación inicial y hasta la educación superior se encontraban matriculados a mediados de 2017 alrededor de 1.1 millones de niños, niñas, adolescentes y jóvenes en casi 14,000 escuelas o planteles predominantemente públicos. De éstos, el $20 \%$ corresponde a alumnos de educación media superior y superior. En la actualidad las Tecnologías de la Información y Comunicación (TIC) en el ámbito educativo han sido potencialmente benéficos en países desarrollados, no siendo iguales en países en desarrollo, generando sustanciales diferencias sociales y tecnológicas.

Las localidades rurales e indígenas se enfrentan a la falta de infraestructura, deficiencias en los servicios de conectividad a Internet, poca capacidad de compra de los dispositivos computacionales y carencia respecto a la habilitación digital limitando su aprovechamiento. En este panorama la expansión de la conectividad en beneficio de sectores de la población de bajos recursos trae consigo nuevas destrezas de alfabetismo y habilidades tecnológicas. (Gómez, 2018).
Las comunidades rurales e indígenas se enfrentan a diversas limitaciones para acceder a las TIC, en el caso del ámbito universitario, esta problemática ha generado que los alumnos en su proceso Enseñanza-Aprendizaje se enfrenten a expectativas y requerimientos para los cuales no están preparados.

En el marco de la estrategia educativa nacional, en la cual se busca garantizar el acceso igualitario a una educación de calidad (INEE, 2018), acorde con las metas específicas referenciadas en la Agenda 2030 propuesta por la Organización de las Naciones Unidas (ONU) para el desarrollo sostenible, la participación de México constituye un requisito indispensable, no sólo en términos ciudadanos y de cohesión social, sino también para el logro de un perfil productivo con mayor valor agregado. Al respecto las Metas Educativas 2021 (OEI, 2010), dejan en claro que, aun considerando las significativas diferencias entre países, o entre distritos y regiones en el interior de cada país, hay importantes problemas comunes, entre ellos se destacan algunas cuestiones relativas a la debilidad de los estados para intervenir, los desequilibrios entre la educación pública y privada, o los problemas de deserción y exclusión, asociados a los impactos que las transformaciones y las variabilidades sociales tienen en el contexto escolar (OEI, 2014).

\section{Análisis conceptual sobre los Nativos Digitales}

La experiencia y el aprendizaje permiten a la gente apropiarse de la tecnología, en ambos casos, proporciona ventajas evidentes a los nativos digitales, sin embargo, en el caso de los "Nativos Digitales Rurales" sus características socioculturales y económicas generan limitaciones y por lo general amplían la brecha digital. En este sentido, conceptos como los de Nativos e Inmigrantes Digitales adquieren un nuevo significado. Acorde con Freire (2008) no son categorías estancas, demográficas o sociales, son fases de un proceso de aprendizaje y desarrollo personal.

Según Palfrey y Gasser (2008), identifican a una nueva generación de jóvenes nacidos en la década de 1980, quienes han crecido inmersos con las tecnologías digitales como parte de su vida cotidiana. 
Se asume que estos jóvenes han crecido alrededor del desarrollo de las computadoras y el Internet, y que por lo tanto tienen aptitudes naturales en el uso de nuevas tecnologías. Con base en lo anterior, se podría considerar que las personas mayores se caracterizan por carecer de esta capacidad natural que conlleva haber crecido rodeado de constantes avances tecnológicos. En el ámbito educativo a este tipo de jóvenes se les adjudican características específicas de aprendizaje demandando rápido acceso a la información, inmediatas recompensas a sus acciones, impaciencia con el pensamiento lineal y capacidad para realizar múltiples tareas (Prensky, 2010). Prensky, indica las características de los Nativos Digitales:

- $\quad \begin{aligned} & \text { Quieren recibir la información de forma } \\ & \text { agil e inmediata. }\end{aligned}$
- Se sienten atraídos por multitareas y
procesos paralelos.
- $\quad$ Prefieren los gráficos a los textos.
$-\quad$ Se inclinan por los accesos al azar (desde
hipertextos).
Funcionan mejor y rinden más cuando
trabajan en Red.
Tienen la conciencia de que van
progresando, lo cual les reporta
satisfacción y recompensa inmediatas.
Prefieren instruirse de forma lúdica a
embarcarse en el rigor del trabajo
tradicional.

En los últimos años se ha difundido la idea de la existencia de una nueva generación de jóvenes con características comunes como resultado de la constante exposición y uso de herramientas tecnológicas. Sin embargo, es necesario realizar investigaciones que permitan identificar estas características representativas de este grupo de jóvenes. En este caso, se debe analizar la situación de los jóvenes universitarios, quienes han sido catalogados como Nativos Digitales, dando por hecho que por ser actualmente universitario cuenta con los conocimientos necesarios en el uso de herramientas tecnológicas. En el ámbito universitario se ha generalizado la idea de que debido a la edad de los jóvenes al ingresar a la universidad cuentan con competencias tecnológicas y de comunicación idóneas para el desarrollo de sus actividades académicas, esta generalización es debido a que se asume que desde la infancia han interactuado con medios digitales y por lo tanto se garantiza el conocimiento en herramientas digitales.

\section{La Región Mixteca y los Nativos Digitales}

El uso, aplicación e implementación de las herramientas tecnológicas en el ámbito universitario inciden en la educación mediática y por lo tanto deben ser objeto de estudio. La incorporación de herramientas tecnológicas en el proceso Enseñanza-Aprendizaje de los alumnos de la UTM, así como la disponibilidad y accesibilidad de los estudiantes de utilizar estas herramientas coadyuvará a alcanzar el objetivo institucional de ofrecer a los alumnos una educación de calidad (Reyes y Allende, 2019).

En la UTM se han realizado diversas investigaciones relacionadas con las competencias académicas de los alumnos de nuevo ingreso, debido a los índices de bajo rendimiento de los estudiantes, detectado en los primeros semestres de su formación académica. Por lo tanto, ha sido necesario analizar la prospectiva tecnológica de los estudiantes de nuevo ingreso a nivel licenciatura de la UTM. Uno de los objetivos de las investigaciones realizadas ha sido identificar las competencias tecnológicas de los alumnos con las cuales se puedan plantear y/o desarrollar estrategias en el proceso de Enseñanza-Aprendizaje con la finalidad de contribuir con un aprendizaje eficiente y significativo (Allende y Reyes, 2020).

Las estadísticas de permanencia estudiantil de la UTM indican que en las últimas generaciones se ha incrementado el índice de deserción de los alumnos, principalmente en los dos primeros semestres de sus estudios. Se debe reconocer la importancia del uso e implementación de herramientas tecnológicas que apoyen tanto a los profesores como a los estudiantes en el proceso de EnseñanzaAprendizaje en la universidad. Sería recomendable aprovechar la experiencia previa del estudiante, facilitando la adaptabilidad del alumno ante nuevas tecnologías, promoviendo la disponibilidad de aprendizaje con plataformas virtuales, etc., con lo cual se puedan proponer acciones que logren la integración plena del alumno a la UTM (Allende y Reyes, 2020).

El análisis geográfico publicado por el Instituto Nacional de Estadística y Geografía (INEGI, 2018), señala que el uso del Internet es un fenómeno urbano, puesto que el $73.1 \%$ del total de la población urbana son usuarios de este servicio, lo que contrasta con el $40.6 \%$ de la población conectada en zonas rurales.

ALLENDE-HERNÁNDEZ Olivia, REYES-ESPINOZA, Celia Bertha y SÁNCHEZ-PLATAS, Liliana Eneida. Estudio exploratorio de los nativos digitales en el ámbito Universitario de la Región Mixteca. Revista de Gestión Universitaria 2020 
Las tres principales actividades de los usuarios de Internet en 2018 fueron: entretenimiento (90.5\%), comunicación $(90.3 \%)$ y obtención de información (86.9\%).

En mayo del 2020, el INEGI publicó que de la población con estudios universitarios el 96.4\% se conecta a la red, mientras que del grupo de personas con estudios de educación básica se conecta el 59.1\%(INEGI, 2019).

En el caso del estado de Oaxaca, las estadísticas del INEGI muestran que:

- Los hogares con conexión a Internet pasaron del $17.8 \%$ del 2015 al $40.4 \%$ en el 2017.

- Los hogares con computadora se incrementaron del $24.6 \%$ el 2015 al $29.1 \%$ el 2017.

- En el 2019, la relación de acceso a Internet por zona urbano-rural presenta una diferencia de 28.9 puntos porcentuales, ya que los resultados reflejan un $76.6 \%$ en las zonas urbanas y $47.7 \%$ en las rurales. Considerando que en el 2018 se había registrado el 49.4\% en zonas urbanas y el $50.6 \%$ en zonas rurales (INEGI, 2019).

Además, en el caso de la Región Mixteca la Secretaría de Finanzas del Gobierno de Oaxaca publicó en los Planes Regionales de Desarrollo de Oaxaca 2011-2016 Región Mixteca el porcentaje de disponibilidad de Tecnologías de Información y Comunicación (TIC) en las viviendas en el periodo de 20112016 (Gobierno de Oaxaca, 2011). En la Tabla 1 se muestra un listado de la disponibilidad de TIC en las viviendas a nivel nacional, a nivel estatal y a nivel regional del estado de Oaxaca.

\begin{tabular}{|l|r|r|r|}
\hline \multicolumn{1}{|c|}{ Nivel } & Computadora & Telefonía & Internet \\
\hline Nacional & 29.4 & 65.1 & 21.3 \\
\hline Oaxaca & 14.3 & 39.3 & 7.8 \\
\hline Cañada & 3.9 & 10.4 & 1.5 \\
\hline Costa & 10.1 & 32.4 & 5.1 \\
\hline Istmo & 14.6 & 46.3 & 7.5 \\
\hline Mixteca & 9.1 & 25.1 & 3.9 \\
\hline Papaloapan & 11.3 & 38.5 & 6.4 \\
\hline Sierra Norte & 5.0 & 5.2 & 1.2 \\
\hline Sierra Sur & 4.9 & 15.6 & 1.8 \\
\hline Valles & 26 & 62.9 & 15.5 \\
Centrales & & & \\
\hline
\end{tabular}

Tabla 1 Porcentaje de Disponibilidad de TIC en las viviendas

Fuente: Planes Regionales de Desarrollo de Oaxaca 2011-201, Mixteca

\section{Análisis de resultados}

En la presente investigación se ha llevado a cabo una revisión informativa con alumnos de primer semestre de la UTM de las carreras de Ingeniería en Diseño, Ingeniería en Mecatrónica e Ingeniería en Computación.

Del conjunto de alumnos participantes, el $62 \%$ son mujeres. Se debe tomar en cuenta que la participación de los alumnos de la carrera de Ingeniería en Diseño incrementa el porcentaje de representación femenina, debido a que en la UTM estadísticamente ha sido una carrera solicitada principalmente por el género femenino, es decir existe una tendencia de formación histórica.

Considerando el acceso a los bienes y servicios de las zonas urbanas en comparación con la disponibilidad de las zonas rurales, se enfatiza la hipótesis de que los alumnos provenientes de las zonas urbanas tienen mayores conocimientos y experiencia en el uso de herramientas tecnológicas. En el levantamiento de información de la presente investigación, los alumnos han indicado que el $65.6 \%$ provienen de zonas urbanas y el $34.4 \%$ de zonas rurales.

En la encuesta realizada en el 2018, aplicada a 152 alumnos de Ingeniería en Mecatrónica y de la Licenciatura en Ciencias Empresariales, el 43\% de los alumnos de nuevo ingreso registró que provienen de la Región Mixteca. El 52.6\% proviene de zonas urbanas y el $47.3 \%$ de zonas rurales. La comparación de los datos estadísticos es significativa debido a que la mayoría de los alumnos provienen de zonas urbanas, con un nivel socioeconómico superior al de los alumnos de zonas rurales. Por ejemplo, el $85 \%$ de los alumnos de zonas urbanas manifestaron contar con casas habitación donde utilizan entre 6 y 15 focos para el consumo de energía eléctrica. En la Figura 1 se muestra gráficamente el número de habitaciones en los hogares de alumnos provenientes de zonas urbanas. 


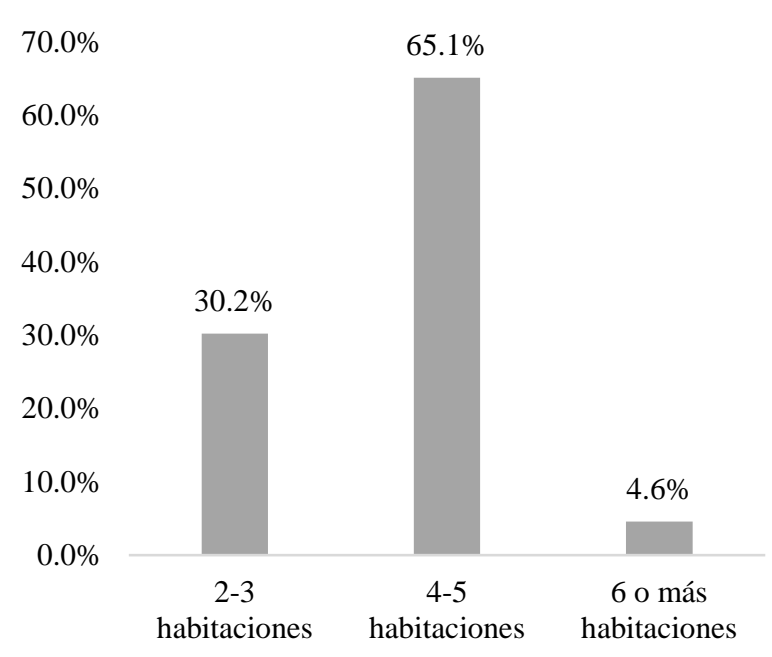

Figura 1 Número de habitaciones en los hogares de alumnos provenientes de zonas urbanas

El $93.4 \%$ de los alumnos han indicado estar registrados en Facebook $\odot$ y el $69.1 \%$ también accede a YouTube $\odot$. En el caso de Instagram es frecuentado por el $59.9 \%$ de los estudiantes. El $75.9 \%$ de los alumnos han indicado que desde el bachillerato han utilizado blogs, videos, correo electrónico, etc. El 27\% de los alumnos ha utilizado aplicaciones educativas desde la secundaria y el $9.9 \%$ desde la primaria.

Los alumnos provenientes de zonas rurales representan el $34.4 \%$ de los alumnos encuestados. En la Figura 2 se muestra el último grado de estudios de los responsables económicos del hogar de donde provienen los estudiantes. Los datos recabados indican que el $33.7 \%$ de los alumnos dependen económicamente de una persona con estudios básicos.

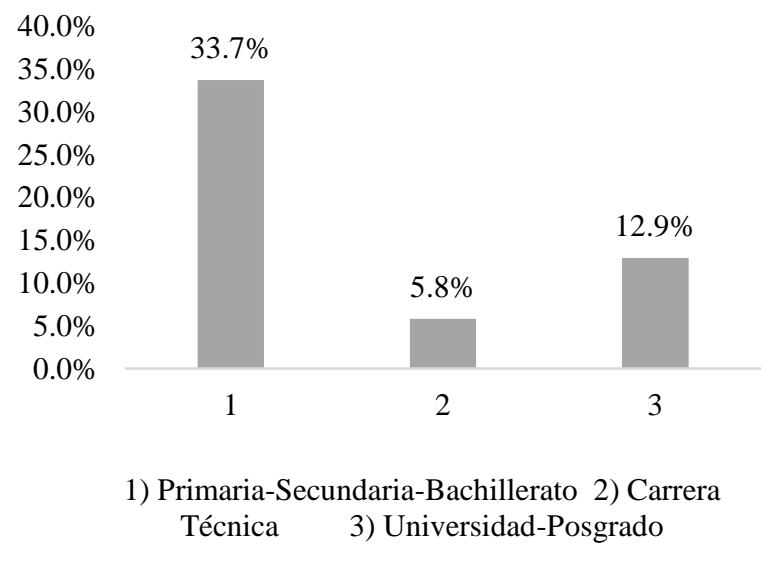

Figura 2 Grado de estudios del responsable económico del hogar de zonas rurales
De los alumnos provenientes de zonas urbanas, la persona que aporta la mayor parte de los ingresos económicos en la familia cuenta con estudios de licenciatura o posgrado, representando el $34.9 \%$ de este grupo de alumnos. En la Figura 3 se muestran los porcentajes del último grado de estudios del responsable económico de los estudiantes provenientes de zonas urbanas.

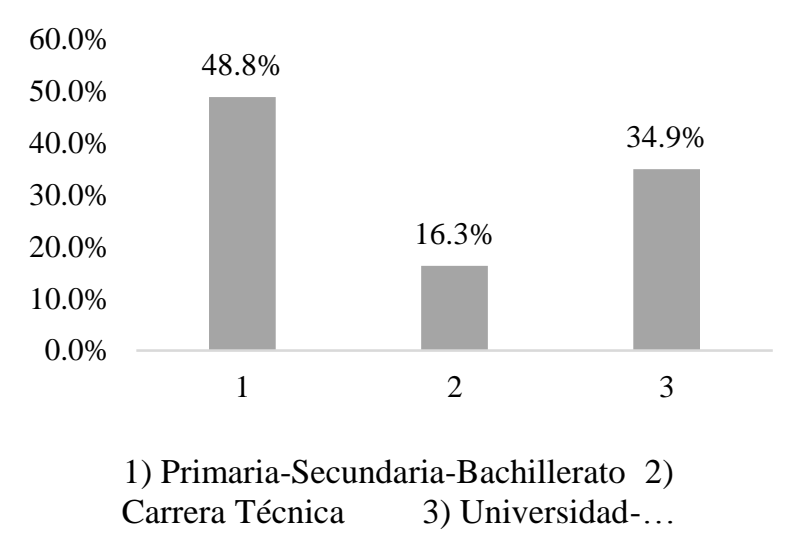

Figura 3 Grado de estudios del responsable económico del hogar de zonas urbanas

Con base en los datos registrados respecto al último grado de estudios del responsable económico del hogar de las zonas urbanas, se puede considerar a estos alumnos con solvencia económica impulsada en la familia. Con este tipo de apoyo económico se le ha permitido al $31.3 \%$ de los alumnos tener acceso a Internet desde la secundaria y al $45.8 \%$ acceder desde la preparatoria.

El $93.8 \%$ de los alumnos expresaron que en su formación académica no recibieron preparación con respecto al uso de Internet o herramientas tecnológicas. A pesar de la ausencia de esta capacitación formal, el $41.2 \%$ de los alumnos se consideran capaces en el uso de mensajería por Internet, el $35.1 \%$ se considera hábil para navegar en Internet e interactuar con buscadores. Sin embargo, indican que son medianamente hábiles para utilizar las plataformas de almacenamiento en línea.

El $27.5 \%$ de los alumnos se considera hábil para realizar podcasting y videocasts. El $28.2 \%$ de los alumnos se considera nada hábil para la manipulación de presentaciones interactivas en red y el $32.1 \%$ se considera nada hábil para utilizar los códigos QR (Quick Response). El 89\% de los alumnos consideran que no tienen ningún nivel de habilidad en lo que respecta a los blogs o wikis. 
Tomando en cuenta la popularidad de las redes sociales, el $38.2 \%$ de los alumnos se considera hábil para encontrar en Facebook $\odot$ información relacionada con las actividades educativas de la Universidad.

La mayoría de los alumnos consideran que no tienen habilidad para resolver problemas técnicos de conexión y para varios de los alumnos no es de su interés este tipo de conocimiento. Se debe considerar la combinación de carreras de los alumnos involucrados en el levantamiento de la información.

El $69.5 \%$ de los alumnos resuelve sus problemas buscando información por sí mismo. Esta situación se reafirma considerando que $61.8 \%$ de los alumnos tienen conexión a Internet desde su casa. Tener acceso a Internet promueve la permanencia en línea, el $51.9 \%$ de los alumnos se mantiene conectado a Internet de entre 1 a 3 horas diarias, para lo cual el $93.9 \%$ lo hace usando el celular y en un segundo lugar usando una computadora portátil.

El $87 \%$ de los alumnos accede a YouTube@ para la realización de sus actividades educativas, y el $42 \%$ usa principalmente WhatsApp@. La mayoría de los alumnos han expresado tener experiencia en el uso de las redes sociales más populares.

\section{Conclusiones}

Se ha demostrado que haber nacido en una era tecnológica no implica poseer competencias digitales. Existen otros factores inmersos en el contexto de los estudiantes de la Región Mixteca que impactan en su competencia y habilidad tecnológica, tal es el caso de los factores socioeconómicos y culturales. Se ha identificado que los alumnos de nuevo ingreso de la UTM tienen diferentes niveles de conocimiento sobre herramientas tecnológicas. A pesar de esta situación, una vez que ingresan a la universidad las exigencias y exceptivas sobre los alumnos se aplican en igualdad de condiciones.

Los alumnos manifiestan su disponibilidad por realizar el trabajo y el estudio necesario para mantener el mismo nivel que el resto de sus compañeros, independientemente de su situación socioeconómica o de las facilidades para el acceso a la tecnología que prevalezca en su vida, así como la disposición al aprendizaje en las competencias digitales.
Es conveniente brindar a los alumnos la oportunidad de subsanar las deficiencias en el uso de herramientas tecnológicas, previo a su ingreso al sistema universitario. Los altos índices de reprobación y deserción de los alumnos en los dos primeros semestres en la UTM, es un indicio de la necesidad de implementar herramientas de apoyo, las cuales les permita garantizar su permanencia en la universidad.

Los alumnos han manifestado gran disponibilidad para realizar las acciones necesarias que les permita subsanar sus deficiencias en conocimientos relacionados con las herramientas tecnológicas. Expresaron que no se consideran a sí mismos como Nativos Digitales, a pesar de poseer varias de las características indicadas por Prensky (Prensky, 2010), que los identifican como tales. Además, han manifestado que son capaces de aprender lo que sea necesario para desarrollar de forma eficiente su proceso de Enseñanza-Aprendizaje.

Por lo tanto, es necesario reconocer que la UTM cuenta con una matrícula de nuevo ingreso con competencia tecnológica heterogénea, en gran medida producto de un contexto sociocultural etnolingüístico, una economía precaria y una compleja orografía propia de la Región Mixteca. Con base en lo anterior se requiere de futuros estudios que permitan un análisis específico del perfil del estudiante de nuevo ingreso, a fin de coadyuvar con procedimientos y programas para el desarrollo y fomento de las habilidades y competencias tecnológicas de los estudiantes.

\section{Referencias}

Allende, O. y Reyes, C. (2020). Technological Foresight of Incoming First-Year University Students in the Mixteca Region. ICERI 2020 13th International Conference of Education, Research and Innovation. IATED. España.

Consejo Nacional de Evaluación de la Política de Desarrollo Social, CONEVAL (2015).

Freire, J. (2008). Las brechas digitales: uso y apropiación. Vida Digital. Recuperado de http://www.soitu.es/soitu/2008/11/07/pieldigital /1226075627_186473.html 
Gobierno del Estado de Oaxaca, (2011). Planes Regionales de Desarrollo de Oaxaca 2011-2016. Mixteca. Recuperado de http://www.finanzasoaxaca.gob.mx/pdf/planes/ planes_regionales/Mixteca/Mixteca.pdf

Gómez, D. (2018). Apropiación social y comunitaria de las TIC en zonas indígenas. UGOB Laboratorio de Innovación. Recuperado de http://u-gob.com/apropiacion-social-ycomunitaria-de-las-tic-en-zonas-indigenas

INEE, (2018). Agenda 2030 para el desarrollo sostenible en educación: retos y prospectiva. Instituto Nacional para la Evaluación de la Educación. México, 2018. Recuperado de https://historico.mejoredu.gob.mx/?s=agenda+2 030\&type $=$ all

INEE, (2019). Panorama Educativo de México. Indicadores del Sistema Educativo Nacional 2018. Educación básica y media superior. Instituto Nacional para la Evaluación de la Educación.

INEGI, (2016), Indicadores Socioeconómicos de los Pueblos Indígenas de México). Recuperado de

https://www.gob.mx/cms/uploads/attachment/fi le/239926/06-cuadro-02.pdf

INEGI, (2018). Encuesta Nacional sobre Disponibilidad y Uso de TIC en Hogares, ENDUTIH. Instituto Nacional de Estadística y Geografía. Recuperado de https://www.inegi.org.mx/temas/ticshogares/

INEGI, (2019). Encuesta Nacional sobre Disponibilidad y Uso de Tecnologías de la Información en los Hogares (ENDUTIH). Recuperado de https://www.inegi.org.mx/contenidos/saladepre nsa/aproposito/2020/eap_internet20.pdf

OEI, (2014). Ciencia, Tecnología e Innovación para el Desarrollo y la Cohesión Social. Organización de los Estados Iberoamericanos para la Educación, la Ciencia y la Cultura. España.

Palfrey, J. y Gasser, U., (2008). Born digital: Understanding the first generation of digital natives. New York: Basic Books.
PNUD, (2016). Programa de las Naciones Unidas para el Desarrollo (PNUD), 2016. Índice de Desarrollo Humano de Hogares e Individuos 2016: el desarrollo humano incluyente. México.

PNUD, (2019). Programa de las Naciones Unidas para el Desarrollo (PNUD), 2019. Informe de Desarrollo Humano Municipal 20102015 Transformando México desde lo local. http://www.mx.undp.org

Prensky, M. (2010). Nativos e Inmigrantes Digitales. Institución Educativa SEK. Albatros, S.L.

Reyes, C. y Allende, O. (2019). Media Education in the University Context in the Mixteca Region. INTED 2019 13th International Technology, Education and Development Conference. España. ISBN 978-84-09-08619-1. 